\title{
Non-Periodicity Challenges in Modelling and Experimental Testing of 3D Woven Composites
}

\author{
Bassam EI Said $^{1 *}$, Stephen R. Hallett ${ }^{1}$ \\ ${ }^{1}$ University of Bristol, United Kingdom. \\ * bassam.elsaid@bristol.ac.uk
}

3D woven composites are made of woven orthogonal layers of yarns (warps/weft) with third direction yarns (binder) woven through thickness. This construction gives 3D composites enhanced mechanical characteristics in the form of high impact resistance, higher toughness and delamination resistance when compared to their 2D counterparts. 3D composites are manufactured from as woven preforms which are then drapped and infused to form the final structure's geometry. During manufacturing the different layers forming the composite preform are constrained from sliding by the binder yarns. Consequently, the internal architecture of 3D woven composites has to deform to conform to the structure geometry. This results in a nonperiodic internal architecture where each location in the structure would experience different
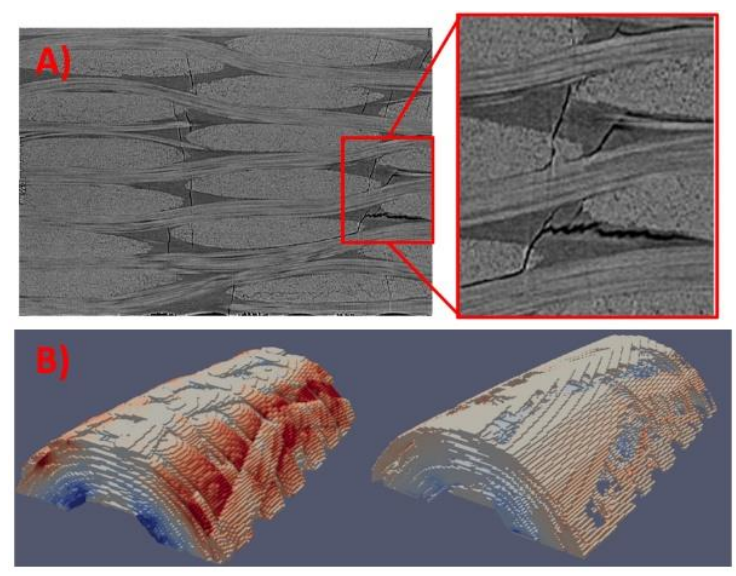

Figure 1. A) CT-Scan of woven composites showing the initiation of damage from local artifacts such as resin pockets. B) High-Fidelity modelling of Woven composites shown the effect of the internal architecture. levels of shearing, bending and compaction[1].

This nonperidocity poses a considerable challenge for conventional modeling and experimental testing techniques. Internal architecture deformations in the form of shearing and compaction are not constant across the structure and consequently each location will have different material articture. Mutliscale modeling techniques which rely on the presence of a repeating unit cell do not provide accurate results due to the absence of an RVE that can be used to predict homogeneous material properties. Similarly, mechanical testing techniques which often rely on testing flat specimens provide results that do not relate to the structures in service. This is due to the fact that the material architecture is different between the materials being tested and the deformed material in an actual structure. Additionaly, specimens following testing standards, such as tension/compression, do not take into account the presence of the woven unit cell and its size compared to the specimen. As a result, the interaction between the woven unit cell edges and the specimen edge leads to high variability in mechanical testing results.

In the this work, by using modeling and mechanical testing of woven composite specimens these nonperiodic challenges are demonstrated, for example, as shown in Figure 1. Additionally, interrupted CT scans are used to demonstrate the progressive nature of damage initiation in 3D composites and how it relates to the internal architecture. Modeling strategies will be disscussed to reduce or eliminate the variability in results originating from the material nonperiodicity. Multiscale modeling strategies based on Vornoi tesslation and domain decompoostion will be disscused [2]. As well, mechnical testing approaches which take the presence of a woven unit cell into account.

\section{References}

[1] B. El Said, S. Green, and S. R. Hallett, "Kinematic modelling of 3D woven fabric deformation for structural scale features," Composites Part A: Applied Science and Manufacturing, vol. 57, pp. 95-107, 2014.

[2] B. El Said, F. Daghia, D. Ivanov, and S. R. Hallett, "An iterative multiscale modelling approach for nonlinear analysis of 3D composites," International Journal of Solids and Structures, vol. 132-133, pp. 42-58, 2018/02/01/ 2018, doi:

https://doi.org/10.1016/j.ijsolstr.2017.08.017. 